\title{
PRODUÇÃO, PURIFICAÇÃo E AUMENTO DA PERFORMANCE DE CICLODEXTRINA GLICOSILTRANSFERASES PARA PRODUÇÃO DE CICLODEXTRINAS
}

\author{
Cristiane Moriwaki, Cassiana Mazzer, Rúbia Pazzetto e Graciette Matioli* \\ Departamento de Farmácia e Farmacologia, Universidade Estadual de Maringá, 87020-900 Maringá - PR, Brasil
}

Recebido em 9/12/08; aceito em 27/5/09; publicado na web em 28/10/09

\begin{abstract}
PRODUCTION, PURIFICATION AND PERFORMANCE IMPROVE OF CYCLODEXTRIN GLYCOSYL TRANSFERASES TO CYCLODEXTRINS PRODUCTION. Biospecific affinity chromatography was used to purify three cyclodextrin glycosyl transferases (CGTases) obtained from microorganisms isolated of soil. The cyclodextrins (CDs) production by CGTases was evaluated using starches from different sources. CDs were measured through the Complexation Theory and by HPLC. CGTase from Bacillus firmus strain $7 \mathrm{~B}$ showed the best production $(30 \mathrm{mmol} / \mathrm{L}$ of $\beta-\mathrm{CD}$ and $4.3 \mathrm{mmol} / \mathrm{L}$ of $\gamma-\mathrm{CD}$ ), and its cultivation conditions were optimized. The maximum enzymatic activity was achieved using lung peptone, soluble starch and agitation speed of $160 \mathrm{rpm}$. Studied CGTases were shown quite interesting for the industrial production of CDs.
\end{abstract}

Keywords: cyclodextrin; CGTase; biospecific affinity chromatography.

\section{INTRODUÇÃO}

Ciclodextrina glicosiltransferase (CGTase, EC 2.4.1.19) é a única enzima capaz de converter amido e substâncias relacionadas em ciclodextrinas (CDs) através de uma reação de transglicosilação intramolecular (ciclização). Também catalisa reações de transglicosilação intermolecular, tais como, acoplamento e desproporcionamento. É uma enzima geralmente extracelular, com fraca atividade de hidrólise e produzida por vários tipos de microrganismos, como Thermoanaerobacterium, Klebsiella oxytoca e algumas espécies de Bacillus, como B. macerans, B. circulans, B. megaterium, B. firmus, B. stearothermophilus e B. lentus. ${ }^{1}$

As CDs são oligossacarídeos cíclicos contendo seis $(\alpha-C D)$, sete $(\beta-C D)$, oito $(\gamma-C D)$ ou mais unidades de glicopiranose unidas por ligações $\alpha-(1,4)$. A molécula de CD é descrita como um cone truncado, cujo interior é hidrofóbico e a superfície hidrofílica. ${ }^{2}$ As características estruturais das mesmas são conhecidas com base em investigações por cristalografia de raios-X, ${ }^{3}$ entretanto, Britto e colaboradore ${ }^{4}$ mostraram que o método da mecânica molecular é o mais indicado para determinação da estrutura geométrica das CDs. Devido esta estrutura, as CDs podem formar complexos de inclusão com várias moléculas orgânicas e inorgânicas, sendo que as propriedades físicas, químicas e/ou biológicas da molécula hóspede podem ser alteradas. As CDs são amplamente utilizadas nas indústrias de alimentos, cosméticos, farmacêutica e na agricultura, atuando como agentes estabilizantes de substâncias voláteis e/ou instáveis, emulsificantes, antioxidantes, na solubilização de compostos insolúveis em água, em sistemas de liberação controlada de drogas, na imobilização de compostos tóxicos para proteção ambiental. ${ }^{3,5-7}$

As iniciativas para aplicação industrial das CDs foram bastante tímidas até a década de 70, devido à baixa disponibilidade e preços inacessíveis. Contudo, o consumo anual de CDs no mundo está crescendo em altas proporções como consequência da intensa pesquisa neste campo no sentido de melhorar a produção, recuperação e purificação deste oligossacarídeo cíclico. ${ }^{3}$ Além disso, a busca por substratos abundantes e de baixo custo tem sido reportada por alguns pesquisadores. ${ }^{8}$

Vários tipos de amido podem ser utilizados como substrato para a CGTase, incluindo amido de milho, batata e mandioca. No Brasil,

\footnotetext{
*e-mail: gmatioli@uem.br
}

o amido de mandioca é uma alternativa interessante como substrato para a produção de CDs, pois, quando comparado a outros tipos de amido, tem um baixo custo de produção e alto rendimento. ${ }^{9}$

Diversos trabalhos visando melhorar o rendimento na produção de CDs têm sido relatados na literatura. Para isto, ensaios são executados com o objetivo de otimizar o cultivo do microrganismo responsável pela produção da CGTase e determinar as condições ideais para se obter a atividade máxima da enzima. ${ }^{8-22}$

Considerando a atual necessidade de disponibilização de CDs para aplicação industrial e a vantagem do substrato de custo reduzido no Brasil, o objetivo deste trabalho foi produzir e purificar CGTases de B. firmus cepa 7B, B. firmus cepa 37 e B. sphaericus cepa 41, bem como confrontar a produção de CDs pelas enzimas purificadas e otimizar as condições de produção da CGTase, buscando gerar um incremento na produção de CDs.

\section{PARTE EXPERIMENTAL}

\section{Produção e purificação das CGTases por cromatografia de afinidade bioespecífica (CAB)}

As enzimas utilizadas neste trabalho foram obtidas a partir de microrganismos alcalofílicos (B. firmus cepa 7B, B. firmus cepa 37 e $B$. sphaericus cepa 41), isolados de solo brasileiro de cultura de aveia, mandioca e soja, respectivamente. Os microrganismos foram semeados em meio sólido, pH 10,3, com a seguinte composição $(\%$ p/V): amido solúvel 1,0; polipeptona 0,5 ; extrato de levedura 0,$5 ; \mathrm{K}_{2} \mathrm{HPO}_{4} 0,1 ; \mathrm{MgSO}_{4} .7 \mathrm{H}_{2} \mathrm{O} 0,02$; corante vermelho do congo 0,$01 ; \mathrm{Na}_{2} \mathrm{CO}_{3} 1,0$ e agar 1,5 . As placas foram incubadas a $37^{\circ} \mathrm{C}$ por $48 \mathrm{~h}$, e as colônias transferidas para frascos de Erlenmeyer de $500 \mathrm{~mL}$, contendo $250 \mathrm{~mL}$ de meio de cultivo líquido de mesma composição do meio sólido, exceto a presença do agar e corante, e o amido solúvel a 2,0\% (p/V). Estes pré-inóculos foram cultivados a $37{ }^{\circ} \mathrm{C}$ por $48 \mathrm{~h}$ com agitação de $120 \mathrm{rpm}$. Em seguida, numa proporção de 5\% (V/V), frascos de Erlenmeyer de $2000 \mathrm{~mL}$, contendo $1000 \mathrm{~mL}$ de meio de cultivo líquido foram inoculados e mantidos a $37^{\circ} \mathrm{C}$ por 5 dias e agitação de $120 \mathrm{rpm}$. As células e o material insolúvel foram removidos por centrifugação $(6000 \mathrm{rpm}$, $15 \mathrm{~min}, 4^{\circ} \mathrm{C}$ ) e o sobrenadante livre de células utilizado como fonte de enzima para purificação. 
As proteínas do sobrenadante livre de células foram precipitadas pela adição de sulfato de amônio com $80 \%$ de saturação a $4{ }^{\circ} \mathrm{C}$ por 48 h. Em seguida, procedeu-se uma vigorosa centrifugação (6000 rpm, $30 \mathrm{~min}, 4^{\circ} \mathrm{C}$ ) e o precipitado proteico foi suspenso em tampão Tris$\mathrm{HCl} 10 \mathrm{mmol} / \mathrm{L}, \mathrm{pH} 8,0$. A CGTase foi purificada por cromatografia de afinidade bioespecífica (CAB) utilizando gel de Sepharose 6B e $\beta$-CD como ligante. ${ }^{23}$

A preparação do suporte cromatográfico para a realização da CAB envolveu duas etapas: a ativação da Sepharose 6B, utilizandose dioxirano (1,4-butanodiol diglicidil éter) e a imobilização do ligante $\beta-C D$ no suporte ativado. Inicialmente a Sepharose $6 \mathrm{~B}$ foi lavada abundantemente com água destilada para eliminar o etanol usado na sua estocagem. Para $20 \mathrm{~g}$ de gel de Sepharose 6B seco por sucção, foram adicionados $20 \mathrm{~mL}$ de 1,4-butanodiol diglicidil éter, $20 \mathrm{~mL}$ de $\mathrm{NaOH} 0,6 \mathrm{~mol} / \mathrm{L} \mathrm{e} 40 \mathrm{mg}$ de $\mathrm{NaBH}_{4}$. A mistura foi agitada mecanicamente por $8 \mathrm{~h}$ a $25^{\circ} \mathrm{C}$. Após esse período, o gel foi lavado com água destilada, para eliminar o 1,4-butanodiol diglicidil éter restante. Terminada a etapa de ativação, foi feita a imobilização do ligante. $\mathrm{O}$ gel obtido na primeira etapa foi estabilizado por $5 \mathrm{~min}$ em uma solução de $\mathrm{NaOH} 0,1 \mathrm{~mol} / \mathrm{L}$ e seco por sucção. Para $20 \mathrm{~g}$ de gel seco, foram adicionados $40 \mathrm{~mL}$ de uma solução de $\beta$-CD $20 \%$ $(\mathrm{p} / \mathrm{V}) \mathrm{em} \mathrm{NaOH} 0,1 \mathrm{~mol} / \mathrm{L}$. Uma nova agitação foi feita por $16 \mathrm{~h}$ a $45^{\circ} \mathrm{C}$. Após o término da segunda etapa, o gel foi lavado com água destilada e, a seguir, com tampão Tris- $\mathrm{HCl} 50 \mathrm{mmol} / \mathrm{L}$, para eliminar as moléculas de $\beta$-CD não imobilizadas. A Figura 1 ilustra as etapas de ativação do gel de Sepharose 6B e imobilização do ligante $\beta$-CD.

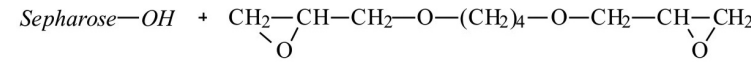

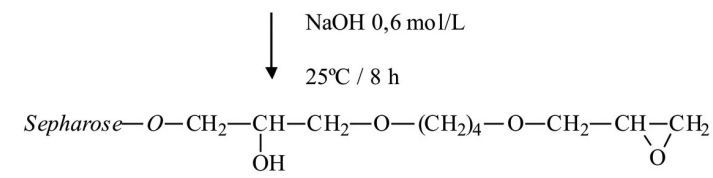

$$
\begin{aligned}
& \beta-\mathrm{CD}-\mathrm{OH} \downarrow \begin{array}{l}
\mathrm{NaOH} 0,1 \mathrm{~mol} / \mathrm{L} \\
45^{\circ} \mathrm{C} / 16 \mathrm{~h}
\end{array}
\end{aligned}
$$$$
\text { Sepharose }-\mathrm{O}-\mathrm{CH}_{2}-\underset{\mathrm{O}}{\mathrm{CH}}-\mathrm{CH}_{2}-\mathrm{O}-\left(\mathrm{CH}_{2}\right)_{4}-\mathrm{O}-\mathrm{CH}_{2}-\underset{\mathrm{OH}}{\mathrm{CH}}-\mathrm{CH}_{2}-\mathrm{O}-\beta-\mathrm{CD}
$$

Figura 1. Esquema de preparação do suporte para a cromatografia de afinidade bioespecífica $(C A B)$

O precipitado proteico suspenso em tampão Tris- $\mathrm{HCl} 10 \mathrm{mmol} / \mathrm{L}$, $\mathrm{pH} 8,0$, foi misturado ao gel ativado, imobilizado e seco e a mistura agitada lentamente por $3 \mathrm{~h}$ a $4{ }^{\circ} \mathrm{C}$.

Para o processo cromatográfico, o gel foi acomodado em uma coluna encamisada acoplada a um banho termostático a $4{ }^{\circ} \mathrm{C}$ e a uma bomba peristáltica, para manter o fluxo ao redor de $0,4 \mathrm{~mL} / \mathrm{min}$.

Na primeira etapa da $\mathrm{CAB}$ o suporte cromatográfico foi estabilizado e lavado com aproximadamente $100 \mathrm{~mL}$ de tampão Tris- $\mathrm{HCl} 50$ $\mathrm{mmol} / \mathrm{L} \mathrm{e} \mathrm{CaCl}_{2} 50 \mathrm{mmol} / \mathrm{L}$ (60\%:40\%). Em seguida, foram aplicados $100 \mathrm{~mL}$ da solução eluente, que consistiu em uma solução de $\beta$-CD $1 \%$ em tampão Tris- $\mathrm{HCl} 50$ mmol/L e CaCl 50 mmol/L (60\%:40\%). O eluente da coluna foi coletado em alíquotas de $2,5 \mathrm{~mL}$. Terminada a passagem do eluente e a coleta das amostras, novamente fez-se a lavagem do suporte cromatográfico.

Para cada fração coletada foi dosada a atividade enzimática e as frações positivas submetidas à ultrafiltração para eliminação da $\beta-C D$ utilizada na eluição e concentração da CGTase. O teor de proteínas foi determinado pelo método de Bradford, ${ }^{24}$ utilizando albumina bovina como padrão.

\section{Avaliação da produção de CDs pelas CGTases purificadas} através de diferentes substratos e pH

A influência da origem do amido sobre a produção de CDs pelas CGTases purificadas de B. firmus cepa 7B, B. firmus cepa 37 e $B$. sphaericus cepa 41 foi testada utilizando soluções de substrato contendo maltodextrina (Dextrin 10, Fluka), amidos de mandioca, como fécula, tapioca e polvilho (cedidos pela Claspar, Maringá PR, Brasil), amidos de milho, arroz e batata (comerciais). Todos esses amidos foram hidrolisados até DE (dextrose equivalente) 10. A hidrólise foi realizada segundo a metodologia de Lima e colaboradores, ${ }^{25}$ utilizando $\alpha$-amilase (Termamyl 120 L, Novo Nordisk, Dinamarca). As condições reacionais do teste de produção de CDs foram: $50{ }^{\circ} \mathrm{C}$, aproximadamente $1 \mathrm{mg} / \mathrm{L}$ de cada enzima pura e as soluções de substrato $10 \%(\mathrm{p} / \mathrm{V})$ dissolvidas em tampão Tris- $\mathrm{HCl} 50$ $\mathrm{mmol} / \mathrm{L}, \mathrm{pH} 8,0$ e $\mathrm{CaCl}_{2} 5 \mathrm{mmol} / \mathrm{L}$, durante $24 \mathrm{~h}$. Amostras foram coletadas em intervalos regulares de tempo e imediatamente inativadas termicamente em banho-maria a $100{ }^{\circ} \mathrm{C}$ por $5 \mathrm{~min}$ para posterior dosagem cromatográfica de $\alpha-C D, \beta-C D$ e $\gamma-C D$ produzidas. Neste ensaio decidiu-se também pela dosagem de $\alpha$-CD e, considerando a dificuldade na dosagem espectrofotométrica desta CD com alaranjado de metila, devido à interferência da $\gamma-\mathrm{CD},{ }^{26}$ recorreu-se à quantificação cromatográfica das CDs por CLAE.

A influência do $\mathrm{pH}$ sob a produção de CDs pela CGTase purificada de B. firmus cepa 7B foi testada utilizando valores de $\mathrm{pH} 6,0$ e 8,0 . Foi utilizado como substrato maltodextrina $10 \%(\mathrm{p} / \mathrm{V})$, aproximadamente $1 \mathrm{mg} / \mathrm{L}$ de enzima pura, $50{ }^{\circ} \mathrm{C}$ durante $24 \mathrm{~h}$. Amostras foram coletadas em intervalos regulares de tempo e imediatamente inativadas termicamente em banho-maria a $100{ }^{\circ} \mathrm{C}$ por 5 min para posterior dosagem espectrofotométrica de $\beta$-CD e $\gamma$-CD produzidas.

\section{Otimização de parâmetros para produção de CGTase}

B. firmus cepa $7 \mathrm{~B}$ foi semeada em meio sólido e incubada a $37^{\circ} \mathrm{C}$ por $48 \mathrm{~h}$. Após este período as colônias foram transferidas para frascos de Erlenmeyer de $250 \mathrm{~mL}$, contendo $100 \mathrm{~mL}$ de um pré-inóculo, até se obter um valor de absorbância de 1,0 a $660 \mathrm{~nm}$. Foram preparados sete meios de cultivo líquido contendo como substrato amido solúvel (padrão), peptona padrão (carne e caseína) e peptonas de diversas origens (carne, fígado, triptona, coração, pulmão, traqueia). Para cada $100 \mathrm{~mL}$ de meio de cultivo líquido contendo as diferentes fontes de peptona, foram transferidos $5 \mathrm{~mL}$ de pré-inóculo e estes mantidos sob agitação de $120 \mathrm{rpm}$ por 5 dias a $37^{\circ} \mathrm{C}$. Ao final do cultivo, os meios foram centrifugados $\left(6000 \mathrm{rpm}, 15 \mathrm{~min}, 4^{\circ} \mathrm{C}\right.$ ) e o sobrenadante livre de células foi utilizado para a determinação da atividade enzimática. As células precipitadas foram utilizadas para determinar densidade óptica a $660 \mathrm{~nm}$.

O mesmo ensaio foi realizado utilizando peptona padrão (carne e caseína) e seis diferentes substratos, como dextrina, amido de milho, amido de mandioca, amido de arroz, amido de batata e amido solúvel (padrão).

Por último, um ensaio utilizando peptona padrão (caseína e carne), amido solúvel (padrão) e diferentes rotações (80, 120, 160 e $200 \mathrm{rpm}$ ) foi realizado e foram determinadas a atividade enzimática e a densidade óptica.

\section{Métodos analíticos}

\section{Determinação espectrofotométrica de $\beta-C D$}

A determinação da concentração de $\beta$-CD utilizando o método de dosagem espectrofotométrico com fenolftaleína (PHE) baseia-se na formação de um complexo entre o corante e a molécula de $\beta-C D$, que causa redução da absorção colorimétrica da solução no comprimento 
de onda de $550 \mathrm{~nm}$. O descoloramento da solução, devido à complexação das moléculas de $\alpha$-CD, $\beta$-CD e $\gamma$-CD com a PHE, é muito mais marcante para a $\beta$-CD sendo, portanto, este método preferencialmente utilizado para a determinação desta CD. Quando a forma ionizada da PHE (vermelha) é encapsulada no interior da $\beta-C D$, este corante é forçado a se manter na sua estrutura lactonada (incolor). Portanto, como o complexo $\beta$-CD-PHE possui uma absorbância diferente da PHE livre, é possível quantificar a $\beta$-CD presente na amostra. ${ }^{27,28}$

Uma curva analítica foi construída conforme segue. Preparou-se uma solução estoque de PHE $3 \mathrm{mmol} / \mathrm{L}$, pesando-se $0,4775 \mathrm{~g}$ de PHE e dissolvendo-se em $500 \mathrm{~mL}$ de etanol 95\%. Foi preparada uma solução tampão carbonato $0,6 \mathrm{~mol} / \mathrm{L}$, pesando-se $50,88 \mathrm{~g}$ de carbonato de sódio e 10,08 g de bicarbonato de sódio e completando-se o volume final para $500 \mathrm{~mL}$ com água destilada. No momento da dosagem foi preparada uma solução de trabalho de PHE, misturando-se $2 \mathrm{~mL}$ da solução estoque de PHE $3 \mathrm{mmol} / \mathrm{L}, 20 \mathrm{~mL}$ de solução tampão carbonato $0,6 \mathrm{~mol} / \mathrm{L}$ e completando-se o volume final para $100 \mathrm{~mL}$ com água destilada.

A solução de $\beta$-CD $1 \mathrm{mmol} / \mathrm{L}$ foi preparada dissolvendo-se 0,1135 $\mathrm{g}$ de $\beta$-CD e completando-se o volume final para $100 \mathrm{~mL}$ com água destilada, contendo $20 \%$ de solução tampão Tris- $\mathrm{HCl} 50 \mathrm{mmol} / \mathrm{L}$ e $10 \%$ de solução de $\mathrm{CaCl}_{2} 50 \mathrm{mmol} / \mathrm{L}$.

Em 21 tubos de ensaio foram colocadas concentrações conhecidas de $\beta$-CD, na faixa de 0 a $1 \mathrm{mmol} / \mathrm{L}$. A diluição foi feita com água tamponada. A dosagem de $\beta$-CD foi realizada misturando-se $0,5 \mathrm{~mL}$ da amostra que continha a $\beta$-CD numa concentração conhecida, com $2,5 \mathrm{~mL}$ da solução trabalho de PHE. A leitura foi efetuada utilizando espectrofotômetro Tecnal SP 1105 a $550 \mathrm{~nm}$ e o branco correspondia à água destilada.

Considerando que a relação entre a absorbância e a concentração de $\beta$-CD somente permanece linear para concentrações até 0,4 $\mathrm{mmol} / \mathrm{L}$, fez-se necessário o uso da teoria da complexação, ${ }^{21,29}$ que fornece a relação não linear entre a absorbância e a concentração de $\beta$-CD. Nesta teoria, assume-se a formação de um complexo com apenas uma molécula de PHE e uma molécula hospedeira ( $\beta-C D)$, e trata-se a formação do complexo como uma reação reversível que está em equilíbrio na solução de dosagem, chegando à definição de uma constante de equilíbrio termodinâmico $\left(K_{\beta-\mathrm{CD}}\right)$. Esta constante foi determinada por regressão não linear da Equação 1, utilizando o método de Quasi-Newton do programa Statistica ${ }^{\circledR}$, para a série de dados obtida na curva padrão.

Portanto, os resultados de absorbância em função da concentração de $\beta$-CD, ajustados pela teoria da complexação, foram calculados de acordo com a Equação 1. Também foram calculados os limites de quantificação e detecção.

$C_{\beta-\mathrm{CD}}=a\left(1-\frac{\mathrm{Abs}_{550}}{\mathrm{Abs}_{0 / 550}}\right)\left(1+\frac{\mathrm{Abs}_{0 / 550}}{K_{\beta-\mathrm{CD}} a \mathrm{Abs}_{550}}\right)$

onde, $C_{\beta-C D}$ é a concentração de $\beta-\mathrm{CD}(\mathrm{mmol} / \mathrm{L}), a$ é a concentração total de PHE na cubeta do ensaio, $\mathrm{Abs}_{550} \mathrm{eAbs}_{0 / 550}$ são as absorbâncias em $550 \mathrm{~nm}$ das amostras e do branco, respectivamente.

\section{Determinação espectrofotométrica de $\gamma-C D$}

A concentração de $\gamma$-CD foi determinada pelo aumento da absorção a $620 \mathrm{~nm}$ de uma solução de verde de bromocresol (VBC) após a complexação com $\gamma$-CD. ${ }^{29} \mathrm{O}$ VBC é um corante específico para determinação de $\gamma-\mathrm{CD}$, uma vez que este corante tem estrutura maior que a cavidade das $\mathrm{CDs}$ menores $(\alpha-\mathrm{CD}$ e $\beta-\mathrm{CD}) .{ }^{30} \mathrm{~A}$ formação do complexo resulta na troca do equilíbrio de protonação, pois as suas formas ácida e básica têm diferentes estabilidades. Considerando que a reação acontece numa solução de $\mathrm{pH} 4,2$, transição ácido-base do corante, a complexação promoverá um aumento no espectro de absorção com mudança na coloração do indicador. ${ }^{26}$
A curva analítica foi construída a partir da preparação de uma solução estoque de VBC $5 \mathrm{mmol} / \mathrm{L}$, pesando-se 1,8 g de VBC e dissolvendo-se em $100 \mathrm{~mL}$ de etanol 96\% e completando-se o volume final para $500 \mathrm{~mL}$ com água destilada. Foi preparada uma solução de ácido cítrico $2 \mathrm{~mol} / \mathrm{L}$, pesando-se $210,14 \mathrm{~g}$ de ácido cítrico mono-hidratado e completando-se o volume final para $500 \mathrm{~mL}$ com água destilada. Também a solução de hidróxido de sódio $2 \mathrm{~mol} / \mathrm{L}$ foi preparada pesando-se $40 \mathrm{~g}$ de $\mathrm{NaOH}$ e completando-se o volume final para $500 \mathrm{~mL}$ com água destilada. No momento da dosagem foi preparada uma solução de trabalho de VBC, misturando-se $10 \mathrm{~mL}$ da solução estoque de VBC $5 \mathrm{mmol} / \mathrm{L}, 10 \mathrm{~mL}$ da solução de ácido cítrico $2 \mathrm{~mol} / \mathrm{L}$ e $5 \mathrm{~mL}$ da solução de $\mathrm{NaOH} 2 \mathrm{~mol} / \mathrm{L}$, completando-se o volume final para $100 \mathrm{~mL}$ com água destilada.

A solução de $\gamma$-CD $2 \mathrm{mmol} / \mathrm{L}$ foi preparada dissolvendo-se 0,2594 g de $\gamma$-CD e completando-se o volume total para $100 \mathrm{~mL}$ com água destilada, contendo $20 \%$ de solução tampão Tris-HCl 50 mmol/L e $10 \%$ de solução de $\mathrm{CaCl}_{2} 50 \mathrm{mmol} / \mathrm{L}$.

Em 21 tubos de ensaio foram colocadas concentrações conhecidas de $\gamma-\mathrm{CD}$, na faixa de 0 a $2 \mathrm{mmol} / \mathrm{L}$. A diluição foi feita com água tamponada. A dosagem de $\gamma$-CD foi realizada misturando-se $0,5 \mathrm{~mL}$ da amostra que continha a $\gamma$-CD numa concentração conhecida, com $2,5 \mathrm{~mL}$ da solução trabalho de VBC. A leitura foi efetuada utilizando espectrofotômetro Tecnal SP 1105 a 620 nm e o branco correspondia à solução do primeiro tubo, que continha uma concentração nula de $\gamma$-CD.

Os resultados de absorbância em função da concentração de $\gamma$-CD foram ajustados por regressão linear e calculados os limites de quantificação e detecção.

\section{Determinação cromatográfica de $C D$ s}

A concentração de $\alpha$-CD, $\beta$-CD e $\gamma$-CD produzidas pelas CGTases purificadas foi determinada por cromatografia líquida de alta eficiência (CLAE) utilizando cromatógrafo líquido CG-480C equipado com detector de índice de refração IR-CG 410, coluna aminopropilsilano SGE com tamanho de partícula $5 \mu \mathrm{m}$, comprimento $25 \mathrm{~cm}$ e diâmetro interno 4,6 mm, acetonitrila e água (65:35) como fase móvel e fluxo de $0,7 \mathrm{~mL} / \mathrm{min}$ à temperatura ambiente. Os padrões e as amostras foram filtrados em membrana de $0,45 \mu \mathrm{m}$. Foram construídas curvas analíticas para $\alpha-C D, \beta-C D$ e $\gamma$-CD com diferentes faixas de concentração. Para a $\alpha$-CD os valores variaram de 1 a $7 \mathrm{mg} / \mathrm{mL}$, para a $\beta$-CD de 1 a $11 \mathrm{mg} / \mathrm{mL}$ e para a $\gamma-C D$ de 1 a $3 \mathrm{mg} / \mathrm{mL}$. Os resultados da área dos respectivos picos em função da concentração de CDs foram ajustados por regressão linear e os limites de quantificação e detecção foram calculados.

As curvas analíticas obtidas tanto pelo método espectrofotométrico quanto cromatográfico foram realizadas em duplicata.

\section{Determinação da atividade enzimática}

A atividade da CGTase foi determinada de acordo com a produção de $\beta$-CD ou $\gamma$-CD pela enzima, que foi medida pelo método espectrofotométrico anteriormente citado.

Uma unidade de atividade (U) corresponde à quantidade de CGTase que libera $1 \mu \mathrm{mol}$ de $\mathrm{CD} / \mathrm{min}$ nas condições reacionais. As condições do ensaio de atividade consistiram na solução de substrato contendo $1 \%(\mathrm{p} / \mathrm{V})$ de maltodextrina em tampão Tris- $\mathrm{HCl} 10 \mathrm{mmol} / \mathrm{L}$ e $\mathrm{CaCl}_{2} 5 \mathrm{mmol} / \mathrm{L}$, a $50{ }^{\circ} \mathrm{C}$. O ponto zero foi obtido colocando-se 1 $\mathrm{mL}$ da enzima em tubo de ensaio com rosca dentro de um banho a $100{ }^{\circ} \mathrm{C}$ por 5 min, para inativação. Após esse tempo, adicionou-se 1 $\mathrm{mL}$ da solução de substrato e o tubo permaneceu por mais $5 \mathrm{~min}$ no banho fervente. $\mathrm{O}$ tubo foi resfriado e conservado em geladeira até $\mathrm{o}$ momento da dosagem. Para os demais pontos, colocou-se $1,0 \mathrm{~mL}$ da solução de substrato em tubo de ensaio com rosca dentro de um banho a $50{ }^{\circ} \mathrm{C}$, aguardou-se a estabilização da temperatura e adicionou-se 
1,0 mL de enzima diluída. Em intervalos de tempos de 5, 10, 15, 20, 25 e $30 \mathrm{~min}$, os tubos foram retirados do banho a $50^{\circ} \mathrm{C}$, transferidos para banho a $100{ }^{\circ} \mathrm{C}$ por $10 \mathrm{~min}$, para parar a reação. Todos os tubos foram resfriados e conservados em geladeira para posterior dosagem espectrofotométrica de $\beta$-CD e $\gamma$-CD produzidas.

O cálculo da atividade foi realizado de acordo com a Equação 2 :

$\mathrm{A}=\frac{\mathrm{K}^{\prime} \times \mathrm{V}_{\mathrm{R}} \times \mathrm{D}}{\mathrm{V}_{\mathrm{E}}}$

onde, A é a atividade enzimática em $\mu \mathrm{mol}$ de $\mathrm{CD} /(\min \times \mathrm{mL})$, $\mathrm{K}$ 'é a inclinação da reta de concentração de CDs em função do tempo (min x mmol/L), $V_{R}$ é o volume total de reação (volume de substrato + volume da enzima), $\mathrm{D}$ é a diluição da enzima e $\mathrm{V}_{\mathrm{E}}$ é o volume da enzima diluída.

\section{RESULTADOS E DISCUSSÃO}

\section{Curvas padrão de CDs}

Os dados da concentração de $\beta$-CD em função da absorbância foram ajustados utilizando a Equação 1, na qual o valor da constante de equilíbrio do complexo $\beta$-CD-PHE $\left(\mathrm{K}_{\beta-\mathrm{CD}}\right)$ foi estimado em 21627 $\pm 85 \mathrm{~L} / \mathrm{mol}$, para um intervalo de confiança de $95 \%$. A substituição das constantes "a" e $\mathrm{K}_{\beta-\mathrm{CD}}$ na Equação 1 resulta na Equação 3, utilizada para a determinação das concentrações de $\beta$-CD nas amostras.

$$
C_{\beta-\mathrm{CD}}=0,3\left(1-\frac{\mathrm{Abs}_{550}}{\mathrm{Abs}_{0 / 550}}\right)\left(1+1,0813 \frac{\mathrm{Abs}_{0 / 550}}{\mathrm{Abs}_{550}}\right) \quad \mathrm{r}^{2}=0,9992
$$

Os limites de quantificação e detecção calculados para a dosagem espectrofotométrica da $\beta$-CD foram de 0,1576 e $0,0472 \mathrm{mmol} / \mathrm{L}$, respectivamente.

A Figura 1S, material suplementar, apresenta os dados experimentais de concentração de $\beta$-CD em função da absorbância, ajustados com a Equação 1 e a Figura $2 \mathrm{~S}$, material suplementar, os dados experimentais de concentração de $\gamma$-CD em função da absorbância, ajustados por regressão linear, na qual se obteve a Equação 4, utilizada para a determinação das concentrações de $\gamma$-CD das amostras.

$C_{\gamma-\mathrm{CD}}=\frac{\left(\mathrm{Abs}_{620}+0,0171\right)}{0,1945}$

$r^{2}=0,9974$

Os limites de quantificação e detecção calculados para a dosagem espectrofotométrica da $\gamma$-CD foram de 0,3598 e 0,1079 $\mathrm{mmol} / \mathrm{L}$, respectivamente.

A dosagem cromatográfica de $\alpha-\mathrm{CD}, \beta-\mathrm{CD}$ e $\gamma-\mathrm{CD}$ por CLAE apresentou curvas analíticas cujas equações, coeficiente de correlação, limite de quantificação e limite de detecção estão abaixo relacionados. $\mathrm{Na}$ Figura 3S, material suplementar, observa-se o cromatograma da solução padrão de $\alpha$-CD, $\beta$-CD e $\gamma$-CD.

Para a determinação da concentração de $\alpha-C D$ :

$C_{\alpha-\mathrm{CD}}=\frac{(\text { Area pico }+64727)}{364073}$

$\mathbf{r}^{2}=0,9992$

Limite de quantificação $=0,5203 \mathrm{mmol} / \mathrm{L}$; Limite de detecção $=0,1561 \mathrm{mmol} / \mathrm{L}$.

Para a determinação da concentração de $\beta$-CD:

$C_{\beta-\mathrm{CD}}=\frac{(\text { Area pico }+117267)}{399214}$

$r^{2}=0,9878$
Limite de quantificação $=1,7385 \mathrm{mmol} / \mathrm{L}$; Limite de detecção $=0,5215 \mathrm{mmol} / \mathrm{L}$.

Para a determinação da concentração de $\gamma$-CD:

$C_{\gamma-\mathrm{CD}}=\frac{(\text { Area pico-31041) }}{3757425} \quad \mathrm{r}^{2}=0.9970$

Limite de quantificação $=0,0542 \mathrm{mmol} / \mathrm{L}$; Limite de detecção $=0,0162 \mathrm{mmol} / \mathrm{L}$.

\section{Comparação das CGTases purificadas através da produção de CDs utilizando diferentes substratos e pH}

O rendimento na produção de $\beta$-CD por meio da utilização das CGTases purificadas de B. firmus cepa 7B, B. firmus cepa 37 e $B$. sphaericus cepa 41 , utilizando como fonte de amido maltodextrina, amido de arroz, amido de batata, amido de milho, polvilho, fécula e tapioca, está demonstrado na Figura 2. A CGTase purificada e obtida a partir de B. firmus cepa 7B foi a enzima que apresentou o melhor desempenho, resultando numa produção maior de $\beta$-CD para todas as fontes de amido. $\mathrm{O}$ amido de milho foi o substrato que proporcionou melhor rendimento na produção de $\beta-\mathrm{CD}$, com exceção da CGTase obtida da cepa 41. Na Figura 3 observa-se o cromatograma da produção de CDs utilizando amido de milho como substrato pela CGTase de B. firmus cepa 7B.

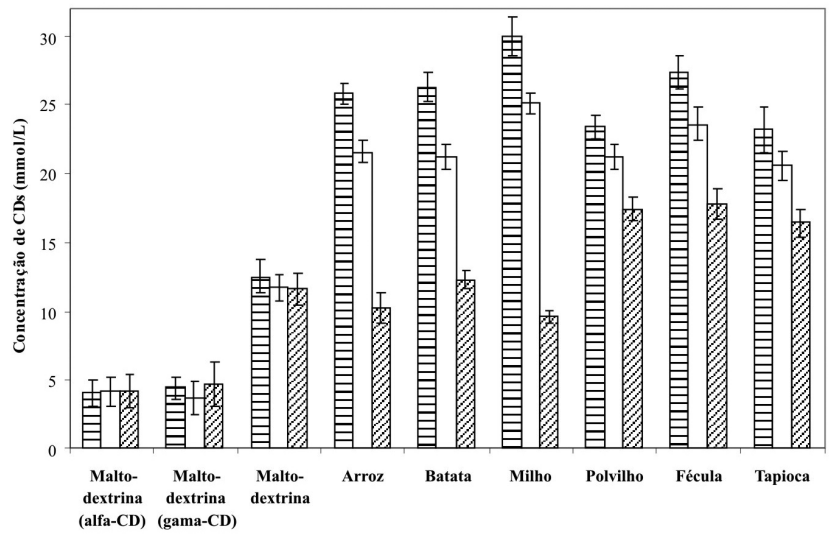

Figura 2. Efeito dos diferentes tipos de amido na produção de $\beta-C D$ pelas CGTases purificadas obtidas a partir de B. firmus cepa $7 B(\equiv)$, B. firmus cepa 37 ( $\square$ ) e B. sphaericus cepa 41 (छ) e da maltodextrina na produção de $\alpha$-CD e $\gamma$-CD. As barras representam o desvio padrão para determinações em duplicata

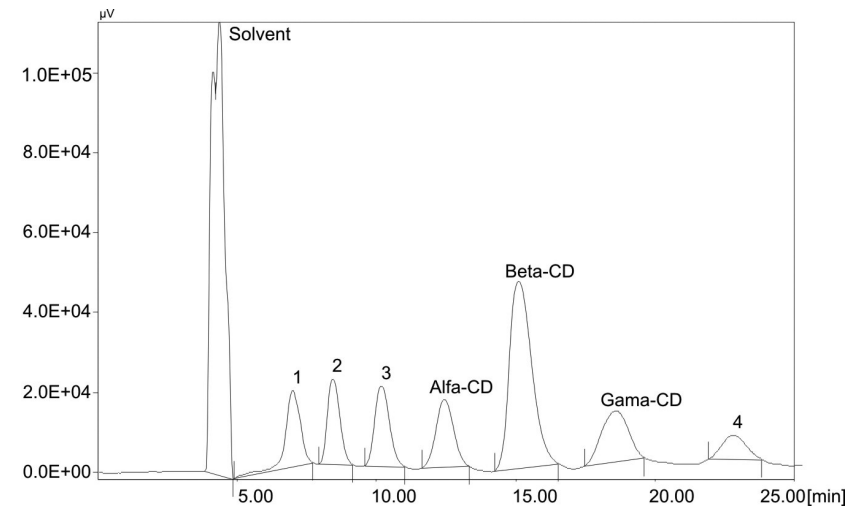

Figura 3. Cromatograma do teste de produção de CDs utilizando amido de milho como substrato e a CGTase purificada de B. firmus cepa $7 B$ após 24 h de reação 
A CGTase de $B$. firmus cepa 7B produziu $30 \mathrm{mmol} / \mathrm{L}$ de $\beta-C D$ a partir do substrato amido de milho, 29,3\% maior quando da utilização do substrato amido de tapioca $(23,2 \mathrm{mmol} / \mathrm{L})$. Também Sian e colaboradores, ${ }^{15}$ para a CGTase do Bacillus sp. G1, observaram a máxima produção de $\beta$-CD com o amido de tapioca (3,2 $\mathrm{mmol} / \mathrm{L})$. Higuti e colaboradores, ${ }^{16}$ utilizando uma CGTase obtida a partir de B. firmus cepa 31 , avaliaram a produção de $\beta-\mathrm{CD}$ a partir de amido de milho Sigma e féculas comerciais. O uso de féculas comerciais de milho $(2,0$ $\mathrm{mmol} / \mathrm{L})$, mandioca $(0,8 \mathrm{mmol} / \mathrm{L})$ e batata $(1,3 \mathrm{mmol} / \mathrm{L})$ diminuiu a produção de $\beta$-CD comparada com amido $\operatorname{Sigma~}(2,3 \mathrm{mmol} / \mathrm{L})$. Isto demonstra que amidos de diferentes origens podem afetar a produção de CDs e este fenômeno é provavelmente devido a diferenças na estrutura física dos grânulos de amido e às suas propriedades. ${ }^{15}$

Embora em nossa pesquisa a fécula de mandioca não tenha demonstrado o melhor rendimento, este produto pode ser um substrato promissor para a produção industrial de $\beta-C D$, em razão da alta disponibilidade desta matéria-prima no Brasil e por apresentar um rendimento muito próximo ao obtido a partir da utilização do amido de milho. Bertolini e colaboradores ${ }^{17}$ também constataram que a produção de CDs usando farelo e fécula de mandioca como substrato é viável, concluindo que o farelo é um substrato adequado quando se visa a produção de $\alpha-C D$, enquanto que a fécula pode ser empregada tanto para a produção de $\alpha$-CD como a de $\beta$-CD.

A produção de $\alpha$-CD e $\gamma$-CD também foi avaliada utilizando maltodextrina como substrato e as CGTases purificadas de B. firmus cepa 7B, B. firmus cepa 37 e B. sphaericus cepa 41 (Figura 2). Para produção de $\alpha-C D$, as três CGTases apresentaram resultados semelhantes, em torno de 4,1 mmol/L. Os melhores rendimentos para a produção de $\gamma$-CD foram obtidos com a utilização das CGTases obtidas a partir de B. firmus cepa 7B e B. sphaericus cepa 41 . Após 24 h de ensaio ambas resultaram na produção de aproximadamente $4,5 \mathrm{mmol} / \mathrm{L}$ de $\gamma$-CD.

De fato, a $\beta$-CD foi o produto predominante para todas as CG-Tases utilizadas neste trabalho. A alta proporção de $\beta$-CD obtida está provavelmente relacionada à origem da enzima. ${ }^{18}$ Estes dados estão em concordância com os resultados obtidos por Sian e colaboradores, ${ }^{15}$ que obtiveram uma maior produção de $\beta$-CD a partir de uma CGTase obtida do microrganismo Bacillus sp. G1.

Considerando que CGTase purificada de B. firmus cepa 7B foi a que apresentou maior rendimento em função das diferentes fontes de substrato, esta enzima foi utilizada na determinação da concentração de $\beta$-CD e $\gamma$-CD produzida em função do $\mathrm{pH}$. Na Figura 4 observa-se que no $\mathrm{pH}$ 8,0 ocorreu a máxima atividade da enzima, sendo que no intervalo de tempo de $24 \mathrm{~h}$ produziu $12,2 \mathrm{mmol} / \mathrm{L}$ de $\beta$-CD. Nesta Figura pode-se observar que em um tempo de produção acima de 24 $\mathrm{h}$, a quantidade de $\beta$-CD provavelmente seria maior.

$\mathrm{O}$ mesmo valor do $\mathrm{pH}$ ótimo não foi obtido para a produção de $\gamma$-CD. Neste caso, a máxima atividade ocorreu em $\mathrm{pH}$ 6,0, fornecendo 4,0 $\mathrm{mmol} / \mathrm{L}$ de $\gamma-\mathrm{CD}$, o que está de acordo com outra CGTase purificada de Bacillus sp. G1 ${ }^{15}$ Cao e colaboradores ${ }^{19}$ observaram um $\mathrm{pH}$ ótimo de 8,5 para a CGTase de Bacillus sp. 7-12 e para Charoenlap e colaboradores ${ }^{9}$ a CGTase do B. circulans atuou melhor na faixa de pH de 4,5 a 5,0. Nos estudos realizados por Higuti e colaboradores, ${ }^{16}$ a atividade da CGTase obtida a partir do B. firmus diminuiu 2,1 vezes quando o $\mathrm{pH}$ inicial foi abaixado de 10,3 para 7,4.

\section{Otimização da produção da CGTase de B. firmus cepa $7 \mathrm{~B}$ através de diferentes fontes de nitrogênio, carbono e velocidades de rotação}

A produção de CGTase por B. firmus cepa 7B foi avaliada empregando-se no meio de cultivo peptonas e amidos de diferentes origens, bem como diversas velocidades de rotação. Os resultados estão expressos na Figura 5A, 5B e 5C, respectivamente.

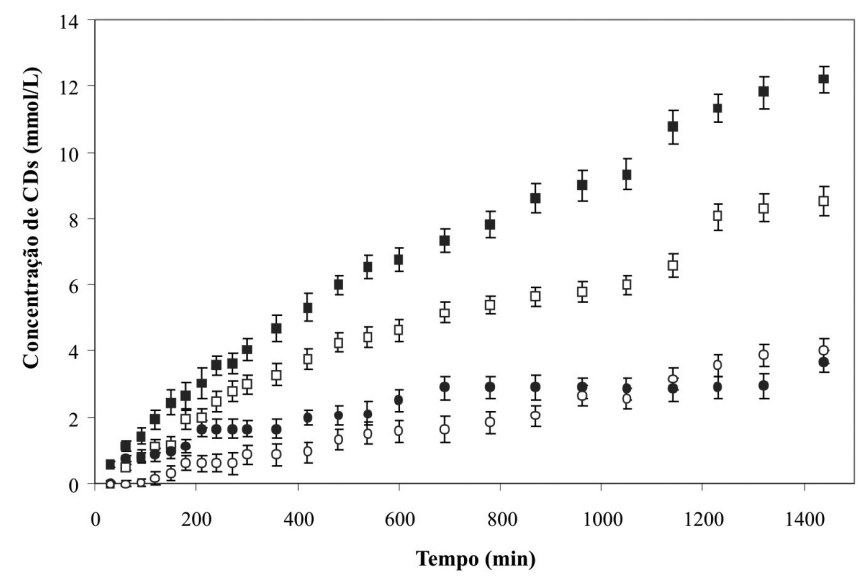

Figura 4. Atividade enzimática para a produção de $\beta-C D$ e $\gamma$-CD em função do $\mathrm{pH}$ por meio da CGTase purificada obtida de B. firmus cepa 7 B. Produção de $\beta$-CD: $\mathrm{pH} 8,0$ e $\square \mathrm{pH}$ 6,0. Produção de $\gamma$-CD: $\bullet \mathrm{pH}$ 8,0 e $\bigcirc \mathrm{pH}$ 6,0. As barras representam o desvio padrão para determinações em duplicata

A maior atividade enzimática encontrada foi de $0,114 \mathrm{U} / \mathrm{mL}$, obtida no sobrenadante livre de células do meio de cultivo que empregou peptona de pulmão como fonte de nitrogênio, apresentando resultados melhores do que aqueles obtidos com a peptona padrão (carne e caseína), que foi de $0,100 \mathrm{U} / \mathrm{mL}$ (Figura 5A). A partir da utilização da peptona de carne foi obtida a maior concentração celular, correspondente à maior densidade óptica, porém, se obteve a menor atividade quando comparada com as outras peptonas testadas. Gawande e colaboradore ${ }^{20}$ testaram a influência de fontes de nitrogênio orgânico na produção da CGTase por B. firmus. Entre as fontes orgânicas testadas, a caseína mostrou-se a mais efetiva, com uma atividade enzimática de $0,48 \mathrm{U} / \mathrm{mL}$, enquanto que a peptona apresentou uma atividade de $0,37 \mathrm{U} / \mathrm{mL}$.

Com relação à influência de diferentes fontes de carbono, a maior atividade obtida foi a partir da utilização do padrão (amido solúvel), seguida pelo amido de arroz e a dextrina (Figura 5B). Gawande e colaboradores $^{20}$ observaram a máxima atividade de CGTase de $B$. firmus com o amido de milho (1,05 U/mL).

Quando foi avaliado o efeito das diferentes velocidades de rotação do meio de cultivo para otimização da produção de CGTase de $B$. firmus cepa 7B, observou-se que ao aumentar a velocidade de rotação também ocorreu um incremento na atividade da enzima, atingindo seu máximo de atividade com 160 rpm (Figura 5C). Igualmente, a CGTase de Bacillus sp. TS1-122 teve um aumento na sua produção (15\%) quando a velocidade de rotação de $200 \mathrm{rpm}$ passou para $400 \mathrm{rpm}$. Em nosso estudo também foi observado que o aumento da velocidade de rotação levou a menores valores de densidade óptica. Segundo Zain e colaboradores, ${ }^{22}$ altas concentrações de biomassa tornam o meio de cultura viscoso, o que leva a uma redução do conteúdo de oxigênio dissolvido e, consequentemente, uma produção reduzida da enzima.

\section{CONCLUSÕES}

A cromatografia de afinidade bioespecífica, utilizada na purificação das CGTases de B. firmus cepa 7B, B. firmus cepa 37 e $B$. sphaericus cepa 41 mostrou-se trabalhosa, contudo, com desempenho bastante efetivo.

Para a produção de $\beta$-CD a CGTase de $B$. firmus cepa 7B apresentou o melhor desempenho e o substrato amido de milho, o melhor rendimento. A concentração de $\beta$-CD obtida com esta enzima e este substrato foi de $30 \mathrm{mmol} / \mathrm{L}$ após $24 \mathrm{~h}$ de ensaio. A fécula de mandioca apresentou um rendimento em CDs próximo ao obtido com 

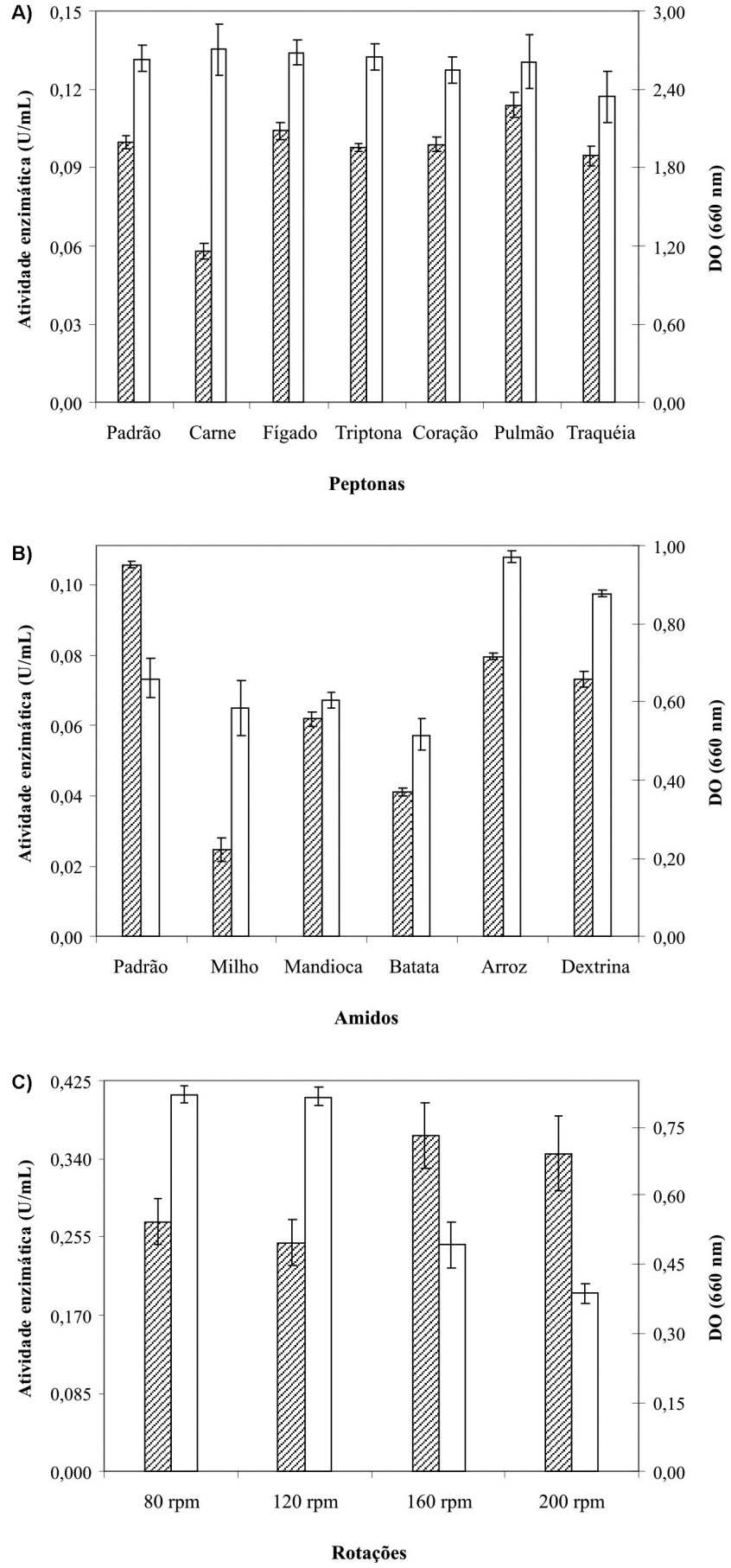

Figura 5. Otimização da produção da CGTase de B. firmus cepa $7 B$. $A$ - Influência das diferentes peptonas. $B$ - Influência dos diferentes amidos. $C$ - Influência das diferentes velocidades de rotação. $₫$ Atividade enzimática $(\mathrm{U} / \mathrm{mL})$ e $\square$ Densidade óptica $(660 \mathrm{~nm})$. As barras representam o desvio padrão para determinações em duplicata

amido de milho, o que torna este produto um substrato promissor para a produção industrial de CDs no Brasil em razão da sua alta disponibilidade, possibilitando a introdução das mesmas no mercado nacional com preços competitivos.

Os valores ótimos de $\mathrm{pH}$ para produção de $\beta$-CD e $\gamma$-CD pela enzima purificada de $B$. firmus cepa 7B foram diferentes. No caso da $\beta$-CD a máxima produção ocorreu em $\mathrm{pH}$ 8,0 e da $\gamma$-CD foi em $\mathrm{pH}$ 6,0 . Este resultado é bastante interessante, possibilitando o direcio- namento da produção de CDs e facilitando as etapas de separação e purificação das mesmas.

As condições de produção de CGTase proveniente de $B$. firmus cepa 7B foram otimizadas e a máxima atividade enzimática foi obtida utilizando peptona de pulmão como fonte de nitrogênio, amido solúvel como fonte de carbono e velocidade de agitação de $160 \mathrm{rpm}$ para produção da enzima.

As CGTases de B. firmus cepa 7B, B. firmus cepa 37 e B. sphaericus cepa 41 mostraram-se bastante interessantes para a produção industrial das CDs. Depois da otimização da produção de enzima, o próximo passo para melhorar a aplicação da CGTase em nível industrial é o estudo da imobilização em diferentes suportes e por diferentes métodos.

\section{MATERIAL SUPLEMENTAR}

As Figuras $1 \mathrm{~S}$ a $3 \mathrm{~S}$ encontram-se disponíveis em http://quimicanova.sbq.org.br, na forma de arquivo PDF, com acesso gratuito.

\section{AGRADECIMENTOS}

À Coordenação de Aperfeiçoamento de Pessoal de Nível Superior (CAPES) pelo suporte financeiro.

\section{REFERÊNCIAS}

1. Biwer, A.; Antranikian, G.; Heinzle, E.; Appl. Microbiol. Biotechnol. 2002, 59, 609 .

2. Singh, M.; Sharma, R.; Banerjee, U. C.; Biotechnol. Adv. 2002, 20, 341.

3. Venturini, C. G.; Nicolini, J.; Machado, C.; Machado, V. G.; Quim. Nova 2008, 31,360 .

4. Britto, M. A. F. O.; Nascimento Jr, C. S.; Santos, H. F.; Quim. Nova 2004, 27, 882 .

5. Del Valle, E. M. M.; Process Biochem. 2004, 39, 1033.

6. Szente, L.; Szejtli, J.; Cyclodextrin Tecnhology, Kluwer Academic Publishers: Dordrecht, 1988.

7. Loftsson, T.; Duchêne, D.; Int. J. Pharm. 2007, 329, 1.

8. Szerman, N.; Schroh, I.; Rossi, A. L.; Rosso, A. M.; Krymkiewicz, N.; Ferrarotti, S. A.; Bioresour. Technol. 2006, 98, 2886.

9. Charoenlap, N.; Dharmsthiti, S.; Sirisansaneeyakul, S.; Lertsiri, S.; Bioresour. Technol. 2004, 92, 49.

10. Matioli, G.; Zanin, G. M.; Guimarães, M. F.; Moraes, F. F.; Appl. Biochem. Biotechnol. 1998, 70-72, 267.

11. Matioli, G.; Zanin, G. M.; Moraes, F. F.; Appl. Biochem. Biotechnol. 2000, 84-86, 955 .

12. Matioli, G.; Zanin, G. M.; Moraes, F. F.; Appl. Biochem. Biotechnol. 2001, 91-93, 643

13. Matioli, G.; Zanin, G. M.; Moraes, F. F.; Appl. Biochem. Biotechnol. 2002, 98-100, 947.

14. Moriwaki, C.; Costa, G. L.; Pazzetto, R.; Zanin, G. M.; Moraes, F. F.; Portilho, M.; Matioli, G.; Process Biochem. 2007, 42, 1384.

15. Sian, H. K.; Said, M.; Hassan, O.; Kamaruddin, K.; Ismail, A. F.; Rahman, R. A.; Mahmood, N. A. N.; Illias, R. M.; Process Biochem. 2004, $40,1101$.

16. Higuti, I. H.; Silva, P. A.; Nascimento, A. J.; Braz. Arch. Biol. Technol. 2004, 47, 135

17. Bertolini, A. C.; Cereda, M. P.; Chuzel, G.; Cienc. Tecnol. Aliment. 1998 , $18,2$.

18. Bovetto, L. J.; Backer, D. P.; Villette, J. R.; Sicard, P. J.; Bouquelet, S. J. L.; Biotechnol. Appl. Biochem. 1992, 15, 48.

19. Cao, X. Jin, Z.; Wang, X.; Chen, F.; Food Res. Int. 2005, 38, 309.

20. Gawande, B. N.; Singh, R. K.; Chauhan, A. K.; Goel, A.; Patkar, A. Y.; Enzyme Microb. Technol. 1998, 22, 288. 
21. Tardioli, P. W.; Zanin, G. M.; Moraes, F. F.; Enzyme Microb. Technol. 2006, 39, 1270.

22. Zain, W. S. W. M.; Illias, R. M.; Salleh, M. M.; Hassan, O.; Rahman, R. A.; Hamid, A. A.; Biochem. Eng. J. 2007, 33, 26.

23. Berna, P.; Moraes, F. F.; Barbotin, J. N.; Thomas, D.; Vijayalaskshmi, M. A.; Adv. Mol. Cell Biol. 1996, 15B, 521.

24. Bradford, M. M.; Anal. Biochem. 1976, 72, 248.

25. Lima, H. O. S.; Moraes, F. F.; Zanin, G. M.; Appl. Biochem. Biotechnol. 1998, 70-72, 789.

26. Barcza, L.; Buvari-Barcza, A.; Carbohydr. Res. 1989, 192, 103.
27. Vikmon, M.; The First International Symposium on Cyclodextrins, Dordrecht, The Netherlands, 1981.

28. Taguchi, K.; J. Am. Chem. Soc. 1986, 108, 2705.

29. Hamon, V.; Moraes, F. F.; Etude Preliminaire a L'immobilisation de L'enzyme CGTase WACKER. In Research Report, Laboratoire de Tecnologie Enzymatique. Compiègne, France: Université de Tecnologie de Compiègne; 1990.

30. Meier, M. M.; Luiz, M. T. B.; Farmer, P. J.; Szpoganicz, B.; J. Inclusion Phenom. Macrocyclic Chem. 2001, 40, 291. 


\section{PRODUÇÃO, PURIFICAÇÃO E AUMENTO DA PERFORMANCE DE CICLODEXTRINA GLICOSILTRANSFERASES PARA PRODUÇÃO DE CICLODEXTRINAS}

Cristiane Moriwaki, Cassiana Mazzer, Rúbia Pazzetto e Graciette Matioli*

Departamento de Farmácia e Farmacologia, Universidade Estadual de Maringá, 87020-900 Maringá - PR, Brasil

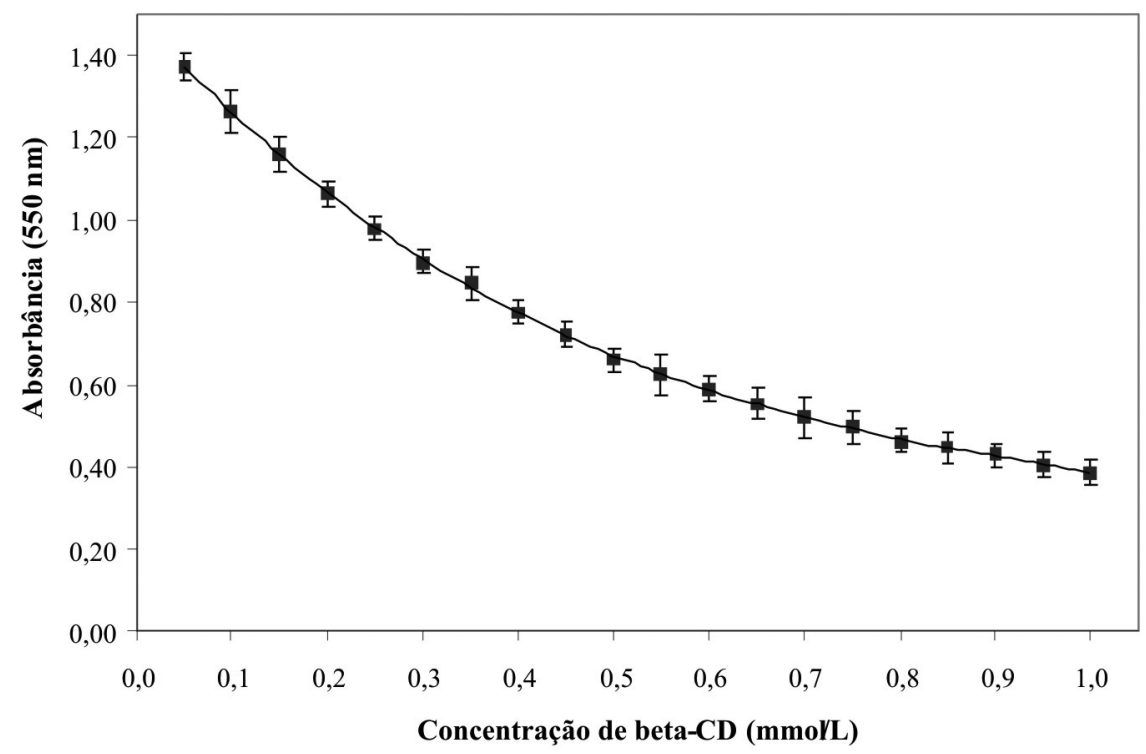

Figura 1S. Curva padrão ajustada pela Teoria da Complexação para dosagem de $\beta$-CD. As barras representam o desvio padrão para determinações em duplicata

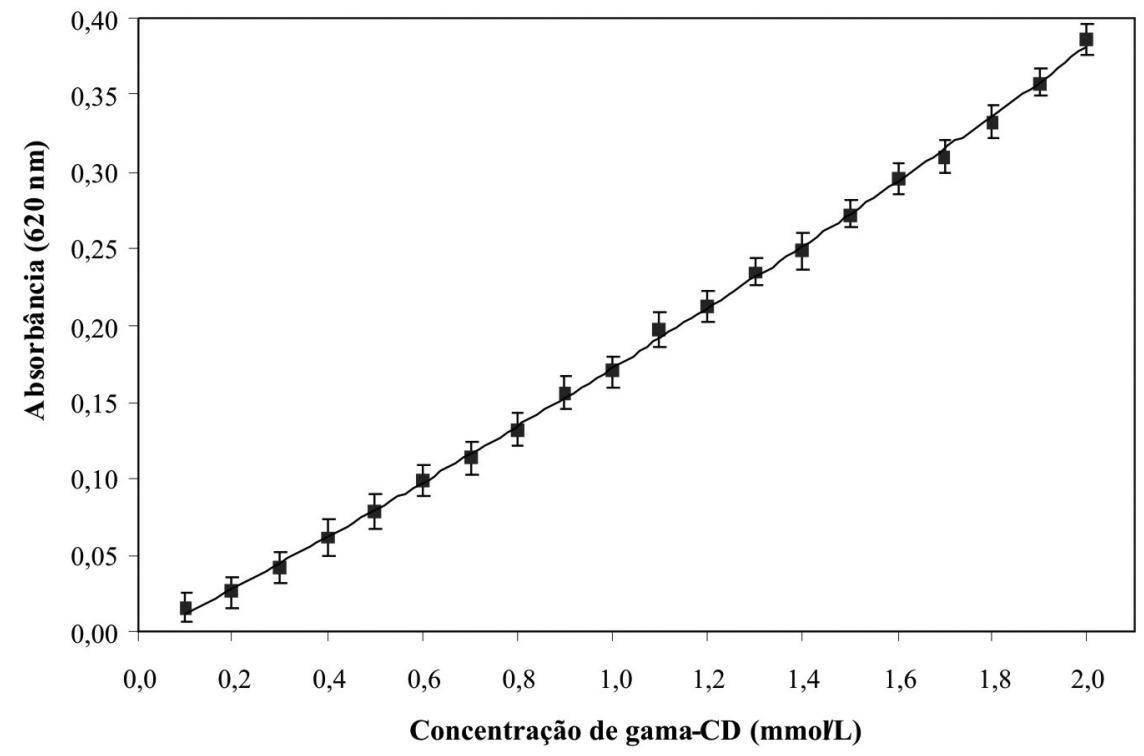

Figura 2S. Curva padrão ajustada por regressão linear para a dosagem de $\gamma$-CD. As barras representam o desvio padrão para determinações em duplicata 


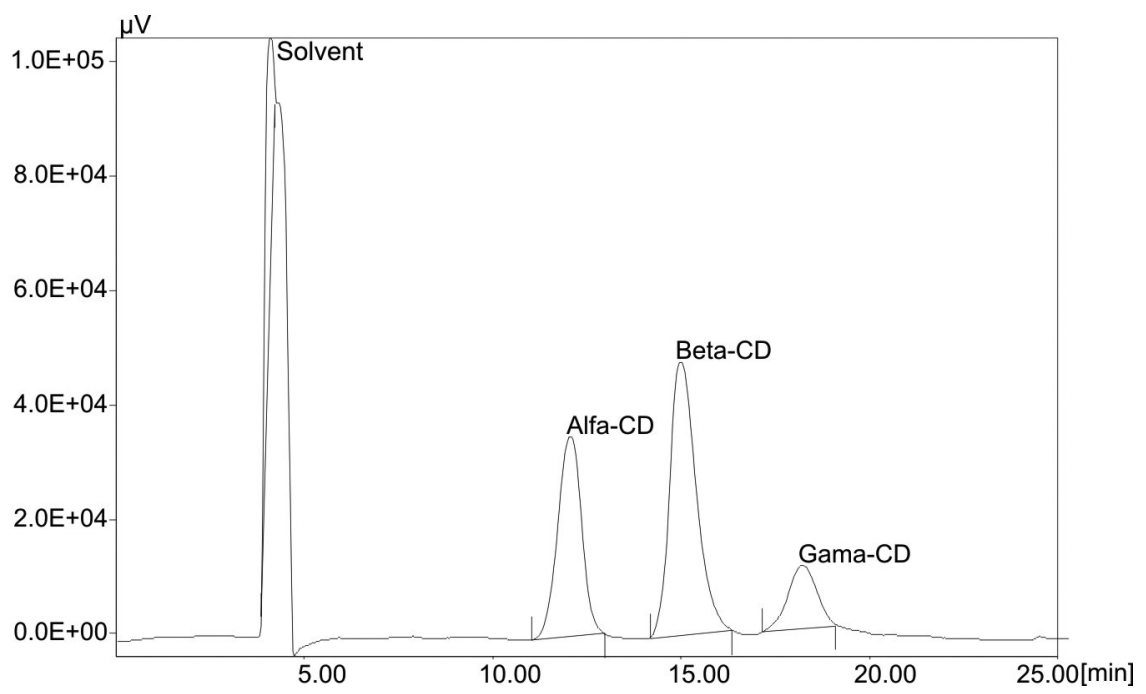

Figura 3S. Cromatograma da solução padrão de $\alpha-C D$, $\beta-C D$ e $\gamma$-CD de 4 , 6 e $2 \mathrm{mg} / \mathrm{mL}$, respectivamente 